\title{
Analysis of Sperm DNA Fragmentation and Aneuploidy in 109 Infertile Patients: Are the Two Parameters Correlated?
}

\author{
Marlea Di Santo, Nicoletta Tarozzi, Marco Nadalini and Andrea Borini*
}

9.Baby-Family and Fertility Center, Via Dante, 15-40125 Bologna, Italy

*Corresponding author: Andrea Borini, 9.Baby-Family and Fertility Center, Via Dante, 15-40125 Bologna, Italy, Tel: 0512867511, E-mail: borini@9puntobaby.it

Received date: 30 July, 2016; Accepted date: 30 August, 2016; Published date: 31 August, 2016

Citation: Di Santo M, Tarozzi N, Nadalini M, et al. Analysis of Sperm DNA Fragmentation and Aneuploidy in 109 Infertile Patients. Are the Two Parameters Correlated?, Gynecol Obstet Case Rep. 2016, 2:2

\section{Abstract}

Purpose: To evaluate the association between sperm DNA integrity and chromosomes aneuploidies in a considerable population of infertile patients undergoing assisted reproductive treatment (109 male) characterized by both normal and abnormal semen parameters.

Methods: In 109 infertile patients with normal and abnormal semen parameters, the assessments of sperm DNA fragmentation by terminal deoxynucleotidyl transferase-mediated dUTP nick-end labelling (TUNEL) and chromosome aneuploidy by fluorescence in situ hybridization (FISH) have been performed on the same semen sample. Sperm samples were collected by masturbation into sterile cups after 3-5 days of sexual abstinence, allowed to liquefy for $30 \mathrm{~min}$ at room temperature and then divided in two aliquots, one processed by double gradient centrifugation (DGC) and used for TUNEL assay, whereas the remained aliquot was left unprocessed and used for FISH test.

Results: The main results indicate a significant positive correlation between sperm chromosome aneuploidies and DNA integrity in patients with abnormal semen parameters $(R=0.516 p=0,000)$ and negative not significant correlation in patients with normal seminal parameters $(R=-0.105, p=0.476)$.

Conclusions: Assuming the positive correlation between the two biomarkers in patients with defective spermatogenesis, high DNA fragmentation index (DFI) could perhaps be indicator of high chromosome aneuploidies level, thus increasing the risk of generating aneuploid embryos. Therefore, a clinical strategy could probably be to integrate also the preimplantation genetic screening (PGS) in ART treatment plan, in addition to the evaluation of sperm DNA fragmentation and/or sperm chromosome aneuploidy, to avoid transfer of chromosomally abnormal embryos.
Keywords: Sperm; DNA fragmentation; Chromosome aneuploidy; Infertility

\section{Introduction}

Infertility is a relatively common condition affecting approximately $15 \%$ of couples worldwide [1,2]. However, the use of assisted reproductive technologies (ART) has greatly increased helping couples to conceive and contributing to the successful birth of over 5 million of babies [3]. For nearly half of these couples, male infertility is notable contributing factor [4]. In particular, male factor infertility has been shown to have a significant, negative effect on the reproductive outcome in ART, despite the application of techniques capable of selecting the best quality spermatozoa among the entire population [5]. One potential interpretation behind this phenomenon might be the introduction of "not healthy" mature spermatozoa during the ART procedure, with defective chromatin organization and/or fragmented DNA, even if apparently they are mobile and morphologically normal [6].

In fact, although semen analysis provides useful information for the evaluation of male infertility, is an imperfect tool for the determination of couple's fertility status and embryo development in ART. It provides no insights into the potentiality of the spermatozoa to fertilize the oocyte and to generate embryos that develop. Nowadays, supplementary molecular tests based on biochemical, proteomic and immunology technical analyses are available to complete the andrological evaluation in fertility clinics laboratories. In this context, biomarkers such as caspase activation, externalization of phosphatidylserine (ePS), alteration of mitochondrial membrane potential, DNA integrity and chromatin organization are the most common tested.

Particularly, it has been largely reported a negative correlation between elevated DNA fragmentation index (DFI) and assisted reproduction outcome $[7,8]$. Although the clinical relevance of sperm DNA fragmentation related to the ART outcome is well known, the causes of sperm DNA damage are still poorly understood [9]. However, some hypotheses have been described in a study by Sakkas et al. [10].

Another important point is the correlation between male infertility and chromosomal abnormalities [11]. Indeed, 
studies reported a higher rate of sperm chromosome aneuploidies in infertile men compared to fertile [9].

Some authors have postulated a potential correlation between sperm chromosomes aneuploidies and DNA integrity. The studies in literature are controversial. Indeed some authors have reported a significant positive correlation between sperm chromosome aneuploidies and DNA integrity in sperm samples from infertile patients [8,9,11-15], suggesting a potential effect of aneuploidy on DNA integrity during sperm maturation. On the contrary, other studies have expressed a different opinion $[16,17]$. Therefore, the question is: sperm DNA fragmentation and aneuploidy, do they really correlate? Unfortunately, insufficient data are available concerning the biological basis of this correlation to bring to a final conclusion.

With our study we aimed to evaluate the relationship between sperm DNA integrity and chromosomes aneuploidies in a considerable population of infertile male patients underwent to ART with either normal or abnormal semen parameters and to bring an additional data to those already reported in the literature.

\section{Materials and Methods}

\section{Patients}

This retrospective study (years 2004-2014) included 109 infertile couples because of recurrent miscarriage and/or repeated ART failure, whom infertility causes are due to male factors or idiopathic infertility. Male partners underwent to sperm DNA fragmentation test by TdT (terminal deoxynucleotidyl transferase)-mediated dUTP nick-end labelling (TUNEL) and chromosome aneuploidy by multi-color fluorescence in situ hybridization (FISH). The tests have been performed on the same semen sample. The mean age of men was $40,8 \pm 6$ (mean \pm SD).

\section{Methods}

Semen sample were collected by masturbation into sterile cups after 3-5 days of sexual abstinence. The semen analysis procedure in this study was performed according to the WHO-2010 guidelines [18]. Semen samples were allowed to liquefy for $30 \mathrm{~min}$ at room temperature and then divided in two aliquots. One aliquot prepared using a discontinuous PureSperm gradient (Nidacon, Gothemberg, Sweden) used for TUNEL assay. Briefly, sperm was layered upon a 40:80\% PureSperm density gradient, processed by centrifuge at $600 \times$ $\mathrm{g}$ for $15 \mathrm{~min}$ and resuspended in $1 \mathrm{~mL}$ of sperm culture medium (PureSperm wash, Nidacon, Gothemberg, Sweden).

Whereas the remained aliquot was left unprocessed and used for FISH test.

\section{DNA fragmentation analysis}

Terminal deoxynucleotidy transferase-mediated deoxyuridine triphosphate (dUTP) in situ DNA nick end labelling (TUNEL) assay was performed on sperm suspension after DGC separation as previously described [19]. Briefly, sperm sample was washed in phosphate-buffered saline (PBS) (Sigma-Aldrich), smeared onto microscope slides, fixed in $4 \%$ paraformaldehyde (Sigma-Aldrich) in PBS for $30 \mathrm{~min}$ at $4^{\circ} \mathrm{C}$ and permeabilized with $0.1 \%$ Triton $\mathrm{X}-100$ in $0.1 \%$ sodium citrate (Sigma-Aldrich). Strand breaks in DNA were detected by TUNEL using a commercially available kit (In situ Cell Death Detection Kit, Fluorescein, Roche) according to the manufacturer's instructions. A negative control was performed for each sample by using fluorescein isothiocyanate (FITC) labelled dUTP without enzyme. The percentage of spermatozoa with fragmented DNA was determined by direct observation of 500 spermatozoa per sample with an epifluorescence microscope (NIKON eclipse $80 \mathrm{i}$ ). The threshold value of $10 \%$ was chosen in line with the previous study of Benchaib et al. [20] performed using the same technique for sperm preparation (DGC separation with PureSperm) and for detection of DNA damage on the sperm suspension obtained in this manner (TUNEL assay and evaluation of positive sperms with epifluorescence microscope).

\section{Sperm chromosomal aneuploidy analysis}

The assessments of sperm aneuploidy were performed by multi-colour fluorescence in situ hybridization (FISH). Briefly, semen samples were washed in phosphate-buffered saline (PBS, pH 7.2, Fischer Scientific International, Pittsburgh, PA, USA) at $400 \mathrm{~g}$ for $5 \mathrm{~min}$, the supernatant discarded and the pellet resuspended in cold methanol: acetic acid (3:1; Sigma, St Louis, MO, USA). Then, $8 \mu \mathrm{L}$ to $10 \mu \mathrm{L}$ drops of fixed sperm were spread on a slide air-dried and eventually stored at $-20 \circ C$ until further processing. The slides were washed twice in $2 \times$ saline sodium citrate (SSC, Fischer Scientific International, Pittsburgh, PA, USA) and dehydrated in an increasing ethanol series (70, 85 and 100\%); then the slides were washed twice in $2 \times$ SSC for $4 \mathrm{~min}$, again dehydrated in an ethanol series $(70,85$ and $100 \%)$ and air-dried. Next, $6 \mu \mathrm{L}$ of the corresponding ready-to-use probe mixture was added to a $15 \times 15$ cover slip. Aneuploidy for chromosome $X, Y, 13,18,21$ was analyzed with $x$ satellite DNA probes by two-colour FISH for chromosomes 13 (spectrum Green) and 21 (spectrum Orange) and three-color FISH for chromosome $X$ (spectrum Green), $Y$ (spectrum Orange) and 18 (spectrum Aqua). Chromosome 18 was also used as an internal autosomal control to distinguish diploidy from sex chromosome disomy. Hybridization was performed overnight into a pre-warmed $37^{\circ} \mathrm{C}$ hybridization chamber (HYBriteTM).

The day after, to eliminate the unspecific hybridization signals, the slide were washed first in $0.4 \times$ SSC solution at 75 o $C$ and then in $2 \times \mathrm{SSC} / 0.1 \% \mathrm{NP} 40$ solution at room temperature. The slides were then counterstained in antifade solution (Vectashield, Vector Laboratories, Burlingame, CA) to the target region with 4', 6-diamidino-2-phenylindole (DAPI, Vysis Inc). In final phase the slides were analysed using a fluorescence microscope (Nikon) equipped with a dual-band filter set for Texas Red/FITC and single-band filter set DAPI, Aqua, FITC and Texas Red. 
Only sperm with intact head and tail morphology and within an area of the slide where consistent hybridization was evident were scored according to previously described criteria [21].

Briefly, sperm were diagnosed as disomic if they presented two or more fluorescent signals for the same chromosome with a size and intensity similar to those detected in normal nuclei; sperm were defined as diploid by the presence of two signals for each of the studied chromosomes in the presence of the sperm tail and an oval head shape; nullisomic sperm were defined by no fluorescent signal being detected for a given chromosome. All signals were separated from each other by at least a single domain. Chromosome abnormality rates were calculated by assessing at least 1500 sperm-patientschromosome probe sets, adhering to strict scoring criteria.

\section{Statistical analyses}

Data analyses were performed using the Statistical Package for the Social Sciences v16.0 (SPSS Inc., Chicago, IL, USA). Means between groups were compared using an independentsamples student's t-test. Correlation between sperm DNA fragmentation and chromosomal aneuploidies were studied using Pearson's correlation coefficient. A p-value of 0.05 was considered significant and highly significant at $p<0.01$ in all circumstances.

\section{Results}

The seminal characteristics of total population (109 patients) are shown in Table $\mathbf{1}$, (the values are reported as mean \pm standard deviation). Based on the seminal parameters the entire population (109 male patients) was divided in two groups, infertile patients with normal semen parameters
(Group $A, n=48$ ) and patients with abnormal semen parameters (Group B, n=61) (WHO criteria 2010) [18]. The differences between the two groups were statistically significant for: sperm concentration $/ \mathrm{mL}\left(62 \times 10^{6}\right.$ vs. $16.4 \times$ $\left.10^{6} \mathrm{p}=0.000\right)$, total sperm number $\left(193 \times 10^{6}\right.$, vs. $40.4 \times 10^{6}$ $p=0.000)$, total motility ( $51 \%$ vs. $34 \% p=0.000)$ and progressive motility ( $45 \%$ vs. $22.4 \% \mathrm{p}=0.000)$, normal morphology ( $23 \%$ vs. $13.1 \% \mathrm{p}=0.000$ ), sperm DNA fragmentation value (TUNEL positive) $(2.5 \%$ vs. $12.3 \% \mathrm{p}=0.000)$ and sperm aneuploidies value (aneuploidy rate) ( $1.4 \%$ vs. $1.9 \% \mathrm{p}=0.000)$.

All the comparisons are reported in Table 2 (the values are reported as mean \pm standard deviation).

Table 1 Seminal characteristics, sperm DNA fragmentation and aneuploidies value of total population $(\mathrm{N}=109)$

\begin{tabular}{|l|l|l|}
\hline & Mean & Std. Dev \\
\hline Sperm concentration $^{\mathrm{a}}$ & 36.7 & 40.4 \\
\hline Total sperm number & 107.6 & 40.4 \\
\hline Total motility $^{\mathrm{b}}$ & 41.8 & 14.1 \\
\hline Progressive motility $^{\mathrm{b}}$ & 32.2 & 15 \\
\hline Normal morphology $^{\mathrm{b}}$ & 17.4 & 13.1 \\
\hline TUNEL $^{\mathrm{b}}$ & 8 & 10 \\
\hline FISH $^{\mathrm{b}}$ & 1.7 & 1.2 \\
\hline Age & 40.8 & 6 \\
\hline $\begin{array}{l}\text { a values express as million/mL } \\
\text { b values express as } \%\end{array}$ & \\
\hline
\end{tabular}

Table 2 Comparison of seminal characteristics, sperm DNA fragmentation and sperm chromosome aneuploidies value between infertile patients with normal semen parameters and infertile patients with abnormal semen parameters. The differences between the two groups are statistically significant for each seminal parameter $(p=0.000)$.

\begin{tabular}{|c|c|c|c|}
\hline & Normal (48 patients) & Abnormal (61 patients) & $\mathbf{p}$ \\
\hline Sperm concentration ${ }^{a}$ & $62.4 \pm 39.2$ & $16.4 \pm 28.1$ & 0.000 \\
\hline Total sperm number & $193 \pm 143$ & $40.4 \pm 70.5$ & 0.000 \\
\hline Total motility ${ }^{b}$ & $51 \pm 6.4$ & $34 \pm 13.7$ & 0.000 \\
\hline Progressive motility ${ }^{b}$ & $45 \pm 7$ & $22.4 \pm 12.2$ & 0.000 \\
\hline Normal morphologyb & $23 \pm 9.2$ & $13.1 \pm 14.2$ & 0.000 \\
\hline TUNEL $^{\mathrm{b}}$ & $2.5 \pm 2.5$ & $12.3 \pm 12.2$ & 0.000 \\
\hline $\mathrm{FISH}^{\mathrm{b}}$ & $1.4 \pm 0.7$ & $1.9 \pm 1.4$ & 0.000 \\
\hline \multicolumn{4}{|c|}{${ }^{a}$ values express as million $/ m L \pm S D$. Dev } \\
\hline
\end{tabular}

The results indicated a positive significant correlation between sperm chromosome aneuploidies and sperm DNA fragmentation considering group $B(R=0.516 ; p=0.000)$ and not significant correlation considering Group $A(R=-0.105, p=0.476)$ (Figure 1). 


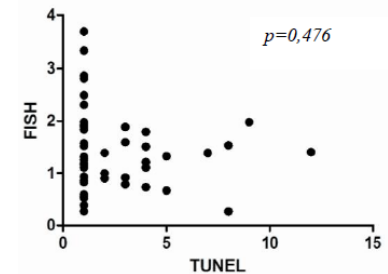

a.

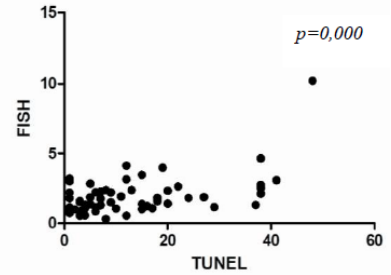

Figure 1 Not significant correlation between sperm chromosome aneuploidies and sperm DNA fragmentation in patients with normal semen parameters $(a)(R=-0.105$, $p=0.476)$ and positive significant correlation in patients with abnormal semen parameters (b) $(R=0.516 p=0.000)$

\section{Discussion}

The introduction of ART has greatly helped couples to conceive babies worldwide. Nevertheless, there are still unexplained causes of infertility that have not been fully understood. It has been widely reported that introduce a "not healthy" spermatozoa during the insemination procedures definitely affects the reproductive outcome [7,22]. Unfortunately, to this day, controversy still persists as to what constitutes the "normal" spermatozoa in semen and any system is guaranteed for the selection of the spermatozoa. Although the application of techniques capable of selecting the best quality spermatozoa, such as the traditional DGC, Swim-Up, or new approaches as magnetic activated cell sorting (MACS) [23], hyaluronan-binding assay (HBA) [24], glass wool filtration, is not possible to have absolute certainty of spermatozoa "health status" at the time of insemination.

The molecular tests introduced in the past years have revolutionized the ART procedures in the laboratories. Among these, the detection of sperm DNA fragmentation and sperm chromosomes aneuploidies are the most studied in literature.

In particular, the association between sperm DNA fragmentation and sperm chromosomes aneuploidy with ART outcome has been well described in literature [22]. On the other hand, remains to shed light on the possible correlation between this two important biomarkers. The data in literature are controversial. This controversy between one study and the other could be explained by the fact that: 1) the findings do not refer to a considerable number of samples; 2) different tests are used to evaluate the sperm DNA integrity; 3) different number of probes to detect chromosomes aneuploidies are analyzed by Fluorescence In Situ Hybridization (FISH); 4) different semen preparation techniques are performed, with or without DGC. With our study we aimed to evaluate the association between sperm DNA integrity and chromosomes aneuploidies in a considerable population of infertile patients undergoing ART (109 male) characterized by both normal and abnormal semen parameters.

The main finding emerging from our study is a significant positive correlation between sperm chromosomes aneuploidy and sperm DNA fragmentation $(R=0.516, p=0.000)$ in patients with abnormal semen parameters (carriers of asthenozoospermia, teratozoospermia, oligozoospermia and oligoasthenoteratozoospermia), whereas no significant correlation in semen samples of patients with normal seminal characteristics was found. This result confirms the previous studies by different authors $[9,12,25,26]$.

\section{Which molecular mechanisms could explain this association?}

During spermatogenesis, programmed cell death (apoptosis) occurs. Thanks to this process genetically abnormal sperm germ cells are eliminated and sperm germ cells in maturation are maintained in a normal ratio. Proliferation of a chromosomally abnormal germ cell (i.e. diploid, haploid spermatogonia) may be blocked through a specific meiotic checkpoints inducing an apoptotic-like process and be associated to DNA fragmentation. Indeed, it has been proposed that aneuploidy could trigger DNA fragmentation, as part of mechanism designed to genetically inactivate a sperm nucleus with an abnormal genomic constitution [9]. However, some of these abnormal gametes escape (abortive apoptosis) from this surveillance mechanism and as a result they are present in the ejaculate $[10,25,27]$. Mature sperm cells with fragmented DNA could correspond to genetically abnormal spermatocytes marked as apoptotic that have not been eliminated but completed the process of meiosis and spermatogenesis (Figure 2). Therefore, it may be speculated that in patients with defective spermatogenesis, the phenomena of abortive apoptosis are supposedly more frequent than in patients with normal spermatogenesis; thus the positive correlation between sperm DNA fragmentation and sperm chromosome aneuploidies in these patients could perhaps be on the origin of their infertility [11].

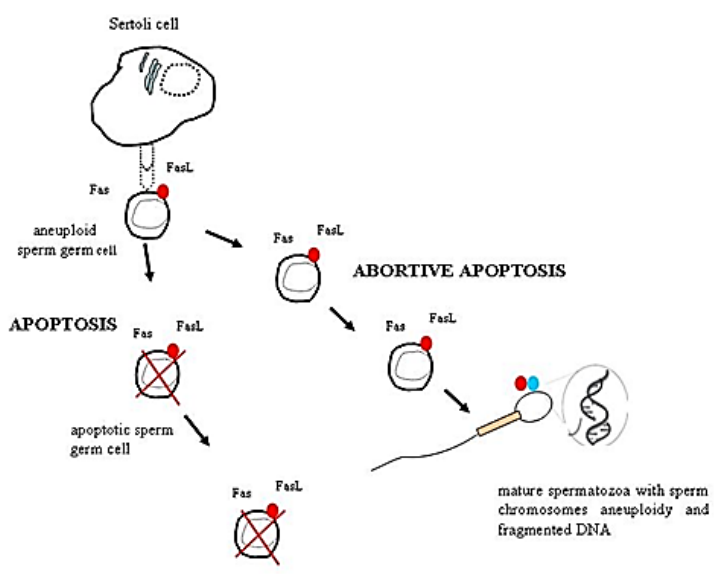

Figure 2 Hypothesis of mechanism of correlation between sperm DNA fragmentation and sperm chromosome aneuploidy in infertile patients with abnormal sperm parameters 
Assuming the positive correlation between the two biomarkers in patients with defective spermatogenesis, obtain high DFI level could be indicator of high chromosome aneuploidies level, thus increasing the risk of generating aneuploid embryos. Therefore, a clinical strategy could probably be to integrate also the preimplantation genetic screening (PGS) in ART treatment plan, in addition to the evaluation of sperm DNA fragmentation and/or sperm chromosome aneuploidy, in order to avoid transfer of chromosomally abnormal embryos. However, since few data in literature regarding this topic are available, further clinical studies are needed before this strategy is applied.

\section{References}

1. Krausz C (2011) Male infertility: pathogenesis and clinical diagnosis. Best Pract Res 25: 271-285.

2. Anawalt BD (2013) Approach to male infertility and induction of spermatogenesis. J Clin Endocrinol Metab 98: 3532-3542.

3. Bloise E, Feuer SK, Rinaudo PF (2014) Comparative intrauterine development and placental function of ART concepti: implications for human reproductive medicine and animal breeding. Hum Reprod 20: 822-839.

4. McLachlan RI, de Kretser DM (2001) Male infertility: the case for continued research. Med J Aust 174: 116-117.

5. Vasan SS (2011) Semen analysis and sperm function tests: How much to test? Indian J Urol 27: 41-48.

6. Sakkas D, Mariethoz E, Manicardi G, Bizzaro D, Bianchi PG, et al. (1999) Origin of DNA damage in ejaculated human spermatozoa. Rev Reprod 4: 31-37.

7. Zhao J, Zhang Q, Wang Y, Li Y (2014) Whether sperm deoxyribonucleic acid fragmentation has an effect on pregnancy and miscarriage after in vitro fertilization/intracytoplasmic sperm injection: a systematic review and meta-analysis. Fertil Steril 102: 998-1005e8.

8. Vendrell X, Ferrer M, Garcia-Mengual E, Munoz P, Trivino JC, et al. (2014) Correlation between aneuploidy, apoptotic markers and DNA fragmentation in spermatozoa from normozoospermic patients. Reprod Biomed Online 28: 492-502.

9. Muriel L, Goyanes V, Segrelles E, Gosalvez J, Alvarez JG, et al. (2007) Increased aneuploidy rate in sperm with fragmented DNA as determined by the sperm chromatin dispersion (SCD) test and FISH analysis. J Androl 28: 38-49.

10. Sakkas D, Alvarez JG (2009) Sperm DNA fragmentation: mechanisms of origin, impact on reproductive outcome, and analysis. Fertil Steril 93: 1027-1036.

11. Enciso M, Alfarawati S, Wells D (2013) Increased numbers of DNA-damaged spermatozoa in samples presenting an elevated rate of numerical chromosome abnormalities. Hum Reprod 28: 1707-1715.

12. Carrell DT, Wilcox AL, Lowy $L$, Peterson $C M$, Jones $K P$, et al. (2003) Elevated sperm chromosome aneuploidy and apoptosis in patients with unexplained recurrent pregnancy loss. Obstet Gynecol 101: 1229-1235.
13. Brahem S, Mehdi M, Elghezal H, Saad A (2011) Analysis of sperm aneuploidies and DNA fragmentation in patients with globozoospermia or with abnormal acrosomes. Urology 77: 1343-1348.

14. Perrin A, Basinko A, Douet-Guilbert N, Gueganic N, Le Bris MJ, et al. (2011) Aneuploidy and DNA fragmentation in sperm of carriers of a constitutional chromosomal abnormality. Cytogenet Genome Res 133: 100-106.

15. Liu CH, Tsao HM, Cheng TC, Wu HM, Huang CC, et al. (2004) DNA fragmentation, mitochondrial dysfunction and chromosomal aneuploidy in the spermatozoa of oligoasthenoteratozoospermic males. J Assist Reprod Genet 21: 119-126.

16. Balasuriya A, Speyer B, Serhal P, Doshi A, Harper JC (2011) Sperm chromatin dispersion test in the assessment of DNA fragmentation and aneuploidy in human spermatozoa. Reprod Biomed Online 22: 428-436.

17. Bronet F, Martinez E, Gaytan M, Linan A, Cernuda D, et al. (2012) Sperm DNA fragmentation index does not correlate with the sperm or embryo aneuploidy rate in recurrent miscarriage or implantation failure patients. Hum Reprod (Oxford, England) 27: 1922-1929.

18. WHO: World health Organization DoRHaR. WHO Laboratory Manual for the Examination and Processing of Human Semen. Geneva: WHO Press, 2010

19. Borini A, Tarozzi N, Bizzaro D, Bonu MA, Fava L, et al. (2006) Sperm DNA fragmentation: paternal effect on early postimplantation embryo development in ART. Hum Reprod 21: 2876-2881.

20. Benchaib M, Braun V, Lornage J, Hadj S, Salle B, et al. (2003) Sperm DNA fragmentation decreases the pregnancy rate in an assisted reproductive technique. Hum Reprod 18: 1023-1028.

21. Blanco J, Egozcue J, Vidal F (1996) Incidence of chromosome 21 disomy in human spermatozoa as determined by fluorescent insitu hybridization. Hum Reprod 11: 722-726.

22. Tarozzi N, Bizzaro D, Flamigni C, Borini A (2007) Clinical relevance of sperm DNA damage in assisted reproduction. Reprod Biomed Online 14: 746-757.

23. Nadalini M, Tarozzi N, Di Santo M, Borini A. Annexin V (2014) Magnetic-activated cell sorting versus swim-up for the selection of human sperm in ART: is the new approach better then the traditional one? J Assist Reprod Genet 31: 1045-1051

24. Tarozzi N, Nadalini M, Bizzaro D, Serrao L, Fava L, et al. (2009) Sperm hyaluronan-binding assay: clinical value in conventional IVF under Italian law. Reprod Biomed Online 19: 35-43.

25. Perrin A, Louanjli N, Ziane $Y$, Louanjli T, Le Roy C, et al. (2011) Study of aneuploidy and DNA fragmentation in gametes of patients with severe teratozoospermia. Reprod Biomed Online 22: $148-154$

26. Brahem S, Mehdi M, Elghezal H, Saad A (2012) Study of aneuploidy rate and sperm DNA fragmentation in large-headed, multiple-tailed spermatozoa. Andrologia 44: 130-135.

27. Brugnon F, Janny L, Communal Y, Darcha C, Szczepaniak C, et al. (2010) Apoptosis and meiotic segregation in ejaculated sperm from Robertsonian translocation carrier patients. Hum Reprod 25: 1631-1642. 